\title{
INHERITANCE PROPERTIES FOR LIPSCHITZ-METRIZABLE FRÖLICHER GROUPS
}

\author{
JOSEF TEICHMANN
}

\begin{abstract}
Frölicher groups, where the notion of smooth map makes sense, are introduced. On Frölicher groups we can formulate the concept of Lipschitz metrics. The resulting setting of Frölicher-Lie groups can be compared to generalized Lie groups in the sense of Hideki Omori. Furthermore Lipschitz-metrics on Frölicher groups allow to prove convergence of approximation schemes for differential equations on Lie groups. We prove several inheritance properties for Lipschitz metrics.
\end{abstract}

\section{INTRODUCTION}

Lipschitz-metrizable groups have been introduced in [6] to show that regularity of Lie groups (see [1] for all necessary details on Lie groups) is closely connected to some approximation procedures possible on Lie groups. The convergence of these approximation schemes is guaranteed by Lipschitz metrics.

In the work of Hideki Omori et al. the beautiful framework of strong ILB-Lie groups is provided (see [2] for example), where the problem of regularity is solved by analytic assumption on the group-multiplication in the charts. The advantage of Lipschitz-metrizable groups is that the notion is "inner", i.e. formulated on the Lie group itself without charts. All strong $I L B$-groups are Lipschitz-metrizable regular groups (see [6], Corollary 2.10). Given a Lipschitz metrizable Lie group we can - by approximation schemes - characterize the existence of exponential and evolution maps. This can also be applied to solve more general equations as stochastic differential equations on Lipschitz-metrizable Lie groups.

In this note we motivate the method of Lipschitz metrics and investigate roughly the inheritance properties of Lipschitz-metrizable Frölicher-Lie groups.

Definition 1. A non-empty set $X$, a set of curves $C_{X} \subset M a p(\mathbb{R}, X)$ and a set of mappings $F_{X} \subset \operatorname{Map}(X, \mathbb{R})$ are called a Frölicher space if the following conditions are satisfied:

(1) A map $f: X \rightarrow \mathbb{R}$ belongs to $F_{X}$ if and only if $f \circ c \in C^{\infty}(\mathbb{R}, \mathbb{R})$ for $c \in C_{X}$.

(2) A curve $c: X \rightarrow \mathbb{R}$ belongs to $C_{X}$ if and only if $f \circ c \in C^{\infty}(\mathbb{R}, \mathbb{R})$ for $f \in F_{X}$

Let $X$ be a Frölicher space, then $C_{X}$ is called the set of smooth curves, $F_{X}$ the set of smooth real valued functions. Maps between Frölicher spaces are called smooth if their composition with any smooth curve is smooth. Let $X, Y$ be Frölicher spaces then the set of smooth maps $C^{\infty}(X, Y)$ has a natural structure of a Frölicher space due to the following requirement:

$$
C^{\infty}(X, Y) \stackrel{C(f, c)}{\rightarrow} C^{\infty}(\mathbb{R}, \mathbb{R}) \stackrel{\lambda}{\rightarrow} \mathbb{R}
$$


is a smooth map for $c \in C_{X}, f \in F_{Y}$ and $\lambda \in C^{\infty}(\mathbb{R}, \mathbb{R})^{\prime}$, where $C(f, c)(\phi):=$ $f \circ \phi \circ c$. The Frölicher space structure generated by these smooth real valued maps is the canonical structure on $C^{\infty}(X, Y)$. The topology $c^{\infty} X$ is given by the initial topology with respect to smooth curves (for all necessary details see [1], chapter $\mathrm{V})$.

A Frölicher group is an abstract group $G$ with the structure of a Frölicher space, such that multiplication and inversion are smooth and $c^{\infty} G$ is a topological group. Furthermore we assume that the smooth functions separate points (smoothly Hausdorff, see [1], chapter VI).

\section{LIPSCHITZ-METRIZABLE FRÖLICHER GROUPS}

In the sequel of the article we shall need the following approximation theorem for product integrals. They exist if their approximations lie uniformly on compact sets in bounded sets, for the notions of convenient calculus we refer to [1]. For the approximation theorem and the notion of product integrals see [2], [4] and [6].

Definition 2 (Product integral). Let $A$ be a convenient algebra. Given a smooth curve $X: \mathbb{R} \rightarrow A$ and a smooth mapping $h: \mathbb{R}^{2} \rightarrow A$ with $h(s, 0)=e$ and $\frac{\partial}{\partial t} h(s, 0)=X(s)$, then we define the following finite products of smooth curves

$$
p_{n}(a, t, h):=\prod_{i=0}^{n-1} h\left(a+\frac{(n-i)(t-a)}{n}, \frac{t-a}{n}\right)
$$

for $a, t \in \mathbb{R}$. If $p_{n}$ converges in all derivatives to a smooth curve $c: \mathbb{R} \rightarrow A$, then $c$ is called the product integral of $X$ or $h$ and we write $c(a, t)=\prod_{a}^{t} \exp (X(s) d s)$ or $c(a, t)=: \prod_{a}^{t} h(s, d s)$. The case $h(s, t)=c(t)$ with $p_{n}(0, t, h)=c\left(\frac{t}{n}\right)^{n}$ is referred to as simple product integral.

Theorem 1 (Approximation theorem). Let $A$ be convenient algebra. Given a smooth curve $X: \mathbb{R} \rightarrow A$ and a smooth mapping $h: \mathbb{R}^{3} \rightarrow A$ with $h(u, r, 0)=e$ and $\frac{\partial}{\partial t} h(u, r, 0)=X_{u}(r)$. Suppose that for every fixed $s_{0} \in \mathbb{R}$, there is $t_{0}>s_{0}$ such that $p_{n}(u, s, t, h)$ is bounded in $A$ on compact $(u, s, t)$-sets and for all $n \geq 1$. Then the product integral $\prod_{s}^{t} h(u, r, d r)$ exists and the convergence is Mackey in all derivatives on compact $(u, s, t)$-sets. Furthermore the product integral is the right evolution of $X_{u}$, i.e.

$$
\begin{aligned}
\frac{\partial}{\partial t} \prod_{s}^{t} h(u, r, d r) & =X_{u}(t) \frac{\partial}{\partial t} \prod_{s}^{t} h(u, r, d r), \\
\prod_{s}^{s} h(u, r, d r) & =e .
\end{aligned}
$$

Remark 1. The hypothesis on the product integrals will be referred to as boundedness condition. For the proof see [4], Theorem 2.2.

The definition of product integrals on Frölicher groups $G$ is done in the same spirit as on convenient algebras, see [6] for details. It yields the natural noncommutative extension of integration. 
Definition 3. Let $G$ be a Frölicher group. Given a smooth mapping $h: \mathbb{R}^{2} \rightarrow G$ with $h(s, 0)=e$, then we define the following finite products of smooth curves

$$
p_{n}(s, t, h):=\prod_{i=0}^{n-1} h\left(s+\frac{(n-i)(t-s)}{n}, \frac{t-s}{n}\right)
$$

for $s, t \in \mathbb{R}$. If $p_{n}$ converges in the smooth topology of $G$ uniformly on compact sets to a continuous curve $c: \mathbb{R} \rightarrow G$, then $c$ is called the product integral of $h$ and we write $c(s, t)=: \prod_{s}^{t} h(u, d u)$. If $h(s, t)=c(t)$, then the product integral $p_{n}(0, t, h)=c\left(\frac{t}{n}\right)^{n}$ is called simple product integral.

Remark 2. Here we need the assumption that $c^{\infty} G$ is a topological group, since we want to talk about uniform convergence and completeness of the uniform space $c^{\infty} G$.

The right regular representation $\rho$ of a Frölicher group $G$

$$
\begin{aligned}
\rho: G & \rightarrow L\left(C^{\infty}(G, \mathbb{R})\right) \\
g & \mapsto(f \mapsto f(. g))
\end{aligned}
$$

in the bounded operators on $C^{\infty}(G, \mathbb{R}$ ) is initial and smooth (see [1] for the notion of initial maps). We shall apply this "linearization" in the sense of Lemma 1 several times in the article (see [6] for further details). This is the main link from the existence theorem for evolutions via Trotter approximations to the general case on Frölicher groups.

Lemma 1. Let $G$ be a Frölicher group, then each product $p_{n}(s, t, h)$ and the limit - if it exists - $\prod_{s}^{t} h(u, d u)$ is smooth. The propagation condition

$$
\prod_{t}^{r} h(u, d u) \prod_{s}^{t} h(u, d u)=\prod_{s}^{r} h(u, d u)
$$

is satisfied for all $r, s, t$.

Proof. By the left regular representation $\rho$ on $G$ we get that the product integral

$$
\lim _{n \rightarrow \infty} p_{n}(s, t, \rho \circ h)
$$

exists in $C^{\infty}\left(\mathbb{R}^{2}, L\left(C^{\infty}(G, \mathbb{R})\right)\right)$, since that image of a sequentially compact set under a smooth mapping is in particular bounded in the convenient algebra $L\left(C^{\infty}(G, \mathbb{R})\right)$. The set formed by $p_{n}(s, t, h)$ and $\prod_{s}^{t} h(u, d u)$ on compact $(s, t)$-sets is sequentially compact due to uniform convergence. Consequently we are in the hypotheses of the approximation theorem Theorem 1, which allows the conclusion of smoothness of $\prod_{s}^{t} h(u, d u)$, since

$$
\rho\left(\prod_{s}^{t} h(u, d u)\right)(f)=\lim _{n \rightarrow \infty} p_{n}(s, t, \rho \circ h)(f)
$$

is smooth and we can evaluate the both sides at $e$ for any $f \in C^{\infty}(G, \mathbb{R})$. Therefore - by the definition of Frölicher spaces - we get the desired conclusion for

$$
(s, t) \mapsto f\left(\prod_{s}^{t} h(u, d u)\right)
$$

is smooth. The propagation condition finally follows from the definition of product integrals and the continuity of multiplication. 
Lipschitz-metrizable Frölicher groups are the adequate framework for the application of Trotter approximations.

Definition 4 (Lipschitz-metrizable groups). Let $G$ be a Frölicher group, such that $c^{\infty} G$ is a topological group. $G$ is called Lipschitz-metrizable if there is a family of right invariant halfmetrics $\left\{d_{\alpha}\right\}_{\alpha \in \nless}$ on $G$ with the following properties:

(1) For all sequences $\left\{x_{n}\right\}_{n \in \mathbb{N}}$ :

$$
\forall \alpha \in \Omega: d_{\alpha}\left(x_{k}, x_{l}\right) \rightarrow 0 \Longleftrightarrow\left\{x_{n}\right\}_{n \in \mathbb{N}} \text { is converging in } G \text {. }
$$

(2) For all smooth mappings $c: \mathbb{R}^{2} \rightarrow G$ with $c(s, 0)=e$, there is on each compact $(s, t)$-set a constant $M_{\alpha}$ such that

$$
d_{\alpha}(c(s, t), e)<M_{\alpha} t .
$$

On Lipschitz-metrizable groups we can formulate a condition for the existence of evolution maps. Therefore we need the notion of "touching at a $e$ ". Given $h_{1,2}: \mathbb{R}^{2} \rightarrow G$ with $h_{1,2}(s, 0)=e$, then we say that $h_{1}$ touches $h_{2}$ at $e$ if for each compact $(s, t)$-set there is a constant $M_{\alpha}$ such that

$$
d_{\alpha}\left(h_{1}(s, t), h_{2}(s, t)\right) \leq M_{\alpha} t^{2}
$$

on the given compact set. A "direction" in $G$ is simply given by an equivalence class of curves $c: \mathbb{R} \rightarrow G$ with $c(0)=e$ under the equivalence relation of "touching at $e$ ". We denote the set of equivalence classes by $\mathfrak{g}$. A smooth group $T: \mathbb{R} \rightarrow G$ is a smooth group homomorphism or smooth one-parameter subgroup, a right evolution $d: \mathbb{R}^{2} \rightarrow G$ is a smooth mapping with $d(s, s)=e$, such that $d(s, t) d(r, s)=d(r, t)$ for all $r, s, t \in \mathbb{R}$. Given a smooth curve $c: \mathbb{R} \rightarrow G$ with $c(0)=e$, then $(s, t) \mapsto$ $c(t) c(s)^{-1}$ is a smooth right evolution.

Theorem 2. Let $G$ be a Lipschitz-metrizable Frölicher group, $h: \mathbb{R}^{2} \rightarrow G$ a smooth mapping with $h(s, 0)=e$, a smooth curve $c: \mathbb{R} \rightarrow G$ with $c(0)=e$ such that $h$ touches the right evolution associated to $c$ at $e$, then the product integral $\prod_{0}^{t} h(s, d s)$ exists and equals $c(t)$. The following estimates are valid for the Lipschitz-metrics $d_{\alpha}:$

$$
d_{\alpha}\left(p_{i}\left(s_{3}, t, c\right)\left(s_{1}\right) p_{n}\left(s_{2}, t+s_{2}, c\right)\left(s_{1}\right) c\left(s_{1}, s_{2}, t\right)^{-1} p_{i}\left(s_{3}, t, c\right)\left(s_{1}\right)^{-1}, e\right) \leq M_{\alpha} t^{2}
$$

for all $i, n \in \mathbb{N}_{+}$on compact $\left(s_{1}, s_{2}, s_{3}, t\right)$-sets given the smooth mapping $d: \mathbb{R}^{3} \rightarrow G$ with $c\left(s_{1}, s_{2}, 0\right)=e$.

Theorem 3. Let $G$ be a Lipschitz-metrizable Frölicher group with $c^{\infty} G$ a topological group. For all smooth mappings $c: \mathbb{R}^{3} \rightarrow G$ with $c\left(s_{1}, s_{2}, 0\right)=e$ the following estimate is valid

$$
d_{\alpha}\left(p_{i}\left(s_{3}, t, c\right)\left(s_{1}\right) p_{n}\left(s_{2}, t+s_{2}, c\right)\left(s_{1}\right) c\left(s_{1}, s_{2}, t\right)^{-1} p_{i}\left(s_{3}, t, c\right)\left(s_{1}\right)^{-1}, e\right) \leq M_{\alpha} t^{2}
$$

for all $n \in \mathbb{N}$ on compact $\left(s_{1}, s_{2}, s_{3}, t\right)$-sets. Then all product integrals exist and depend smoothly on smooth parameters.

Remark 3. In the case of convenient Lie groups this condition can be interpreted as condition on the "second derivatives" of the multiplication mapping. Both theorems are proved in [6]. These Theorems could also be applied to obtain "regular" solutions of Stratonovich differential equations on compact (or non-compact) manifolds by product integration of stochastic vector fields. This will be worked out elsewhere. 
Given a Frölicher group $G$, then we define its tangent space $T_{e} G$ in the following way: $\rho: G \rightarrow L\left(C^{\infty}(G, \mathbb{R})\right)$ is smooth, so

$$
T_{e} G:=\left\{\left.\frac{d}{d t}\right|_{t=0} \rho(c(t)) \text { for } c: \mathbb{R} \rightarrow G \text { smooth with } c(0)=e\right\} .
$$

This is a linear subspace of $L\left(C^{\infty}(G, \mathbb{R})\right)$. Given $\Phi: G \rightarrow H$ smooth, then the tangent map $\Phi^{\prime}: T_{e} G \rightarrow T_{e} H$ can be defined in the following way: Given $X \in T_{e} G$, then there is $c: \mathbb{R} \rightarrow G$ with $c(0)=e$ such that $X=\left.\frac{d}{d t}\right|_{t=0} \rho(c(t))$, we define $\left(\Phi^{\prime}(X) \cdot f\right)(h):=\left.\frac{d}{d t}\right|_{t=0} f(\Phi(h c(t)))$ for all $h \in H$ and $f \in C^{\infty}(H, \mathbb{R})$. This does not depend on the representative since $f(h \Phi()$.$) for fixed h \in H$ is a smooth function on $G$ and two representatives $c_{1}$ and $c_{2}$ with $c_{1}(0)=c_{2}(0)=e$ are equal if for all smooth functions $f: G \rightarrow \mathbb{R}$ the equality $\left.\frac{d}{d t}\right|_{t=0} f\left(c_{1}(t)\right)=\left.\frac{d}{d t}\right|_{t=0} f\left(c_{2}(t)\right)$ holds. The tangent map of the conjugation $\operatorname{conj}(g)(h):=g h g^{-1}$ for $g, h \in G$ is denoted by $A d(g)$ and defines a group isomorphism $A d: G \rightarrow \operatorname{Lin}\left(T_{e} G\right)$. Notice that these definitions do not require charts, however, we are interested in more analytic structures on $G$ itself to be able to apply the above linearizations adequately.

Definition 5. A Lipschitz-metrizable Frölicher group $G$ is called Frölicher-Lie group if $\mathfrak{g}$ carries a convenient structure by defining

$$
\begin{aligned}
{\left[c_{1}\right]+\left[c_{2}\right] } & =\left[c_{1} \cdot c_{2}\right], \\
\lambda \cdot[c] & =[c(\lambda .)]
\end{aligned}
$$

with the norm-functions on $\mathfrak{g}$, namely

$$
p_{\alpha}([c]):=\underline{\lim }_{t \downarrow 0} \frac{d_{\alpha}(c(t), e)}{t} .
$$

Furthermore the mapping $s \mapsto[t \mapsto h(s, t)]$ is assumed to be smooth for any smooth mapping $h: \mathbb{R} \rightarrow G$ with $h(s, 0)=e$ and this are all smooth mappings. Finally there is a diffeomorphism

$$
\text { Evol }^{r}: C^{\infty}(\mathbb{R}, \mathfrak{g}) \rightarrow C^{\infty}(\mathbb{R},\{0\} ; G,\{e\})
$$

defined by $\operatorname{Evol}^{r}(s \mapsto[h(s,)])=.\prod_{0}^{t} h(s, d s)$, where $h: \mathbb{R}^{2} \rightarrow G$ a smooth mapping with $h(s, 0)=e$. The inverse is given by $\delta^{r} c$, the right logarithmic derivative. Furthermore we denote $\operatorname{Evol}^{r}(t \mapsto t X)=: \exp (t X)$, the exponential map.

Remark 4. On strong ILB-groups we are given the structure of a Lipschitzmetrizable Frölicher-Lie group, see [6], Corollary 2.10. Due to these properties one can view Lipschitz-metrics on Lie groups as non-abelian generalizations of seminorms on convenient locally convex spaces (which are abelian Lie groups).

Lemma 2. Let $G$ be a Frölicher-Lie group such that $\mathfrak{g}$ carries the above convenient structure, then $\rho: G \rightarrow L\left(C^{\infty}(G, \mathbb{R})\right)$ induces a unique smooth mapping $\rho^{\prime}: \mathfrak{g} \rightarrow L\left(C^{\infty}(G, \mathbb{R})\right)$ with $\left(\rho^{\prime}([c]) \cdot f\right)(g):=\left.\frac{d}{d t}\right|_{t=0} f(g c(t))$.

Proof. Take a curve $c: \mathbb{R} \rightarrow G$ with $c(0)=e$, then $\lim _{n \rightarrow \infty} c\left(\frac{t}{n}\right)^{n}=: T_{t}$ defines a smooth group. $\rho\left(c\left(\frac{t}{n}\right)^{n}\right)=\left[\rho\left(c\left(\frac{t}{n}\right)\right)\right]^{n}$ converges to $\rho\left(T_{t}\right)$ in the convenient topology of $L\left(C^{\infty}(G, \mathbb{R})\right)$, so in particular $f\left(g c\left(\frac{t}{n}\right)^{n}\right) \rightarrow f\left(g T_{t}\right)$ for every $g \in G$ on compact $t$-intervals in any derivative, so

$$
\left.\frac{d}{d t}\right|_{t=0} f(g c(t))=\left.\frac{d}{d t}\right|_{t=0} f\left(g c\left(\frac{t}{n}\right)^{n}\right)=\left.\frac{d}{d t}\right|_{t=0} f\left(g T_{t}\right) .
$$


So $\rho^{\prime}$ is well defined on every equivalence class and if we take a smooth curve $X$ into $\mathfrak{g}$ we see by the exponential map that

$$
\left(\left(\rho^{\prime} \circ X\right)(s) \cdot f\right)(g)=\left.\frac{d}{d t}\right|_{t=0} f(g \exp (t X(s))),
$$

which is smooth.

Given two Frölicher-Lie groups $G$ and $H$ and a smooth homomorphism $\Phi: G \rightarrow$ $H$, then $\phi:=\Phi^{\prime}$ defined via $\Phi^{\prime}([c])=[\Phi \circ c]$ is a smooth linear mapping. Since by regularity every equivalence class $[c]$ contains a smooth group, we see that $\Phi^{\prime}$ is well defined and smooth. If $G$ is a Frölicher-Lie group, then we can define the Lie bracket on $\mathfrak{g}$ : Given $g \in G$, then there the conjugation $\operatorname{conj}(g)$ is a smooth mapping. $\operatorname{conj}(g)^{\prime}=: A d(g)$ is smooth and depends smoothly on $g$. Given $c: \mathbb{R} \rightarrow G$ with $c(0)=e$, then $[[c],[d]]:=\left.\frac{d}{d t}\right|_{t=0} A d(c(t)) \cdot[d]$.

Lemma 3. Let $G$ be a Frölicher-Lie group, then $\delta^{r}(c d)(s)=\delta^{r} c(s)+\operatorname{Ad}(c(s))$. $\delta^{r} d(s)$ for smooth curves $c$ and $d$.

Proof. Remark that $\delta^{r} c$ for a smooth curve $c$ is defined by "touching at $e$ " of $t \mapsto$ $c(t+s) c(s)^{-1}$. $\left[A d(c(s)) \delta^{r} d\right](s)$ is defined via "touching at $e$ " of $t \mapsto \operatorname{conj}(c(s)) d(t+$ $s)(\operatorname{conj}(c(s)) d(s))^{-1}$, so we see immediately

$$
\begin{gathered}
{\left[t \mapsto c(t+s) d(t+s) d(s)^{-1} c(s)^{-1}\right]} \\
=\left[t \mapsto c(t+s) c(s)^{-1} \operatorname{conj}(c(s)) d(t+s)(\operatorname{conj}(c(s)) d(s))^{-1}\right],
\end{gathered}
$$

which yields the formula.

Remark 5. A Lipschitz-metrizable Frölicher group such that the space $\mathfrak{g}$ is a convenient vector space with the above structures, in particular

$C^{\infty}(\mathbb{R}, \mathfrak{g})=\left\{s \mapsto[t \mapsto h(s, t)]\right.$ for $h: \mathbb{R}^{2} \rightarrow G$ a smooth mapping with $\left.h(s, 0)=e\right\}$

is a Frölicher-Lie group if and only if the conditions of Theorem 3 are valid. This is proved by Theorem 2 and Theorem 3. In particular this is true for all Lipschitzmetrizable convenient Lie groups. Notice that further elements of Lie theory can be developed similarly to [1].

\section{INHERITANCE PROPERTIES FOR FrÖLICHER-LIE GROUPS}

A virtual Frölicher-Lie subgroup $H$ of a Frölicher-Lie group $G$ is defined to a subgroup together with $\mathfrak{h}$, a $c^{\infty}$-closed subalgebra of $\mathfrak{g}$ such that $E v l^{r}: C^{\infty}(\mathbb{R}, \mathfrak{h}) \rightarrow$ $C^{\infty}(\mathbb{R},\{0\} ; H,\{e\})$ is a diffeomorphism. A Frölicher-Lie subgroup is a closed, virtual Frölicher-Lie subgroup.

Theorem 4. We obtain the following inheritance properties:

(1) Let $G_{i}$ for $i \in I$ be an arbitrary set of Frölicher groups, then $\prod_{i \in I} G_{i}$ is a Frölicher group.

(2) Let $G$ be a Frölicher-Lie group and $H$ a closed subgroup, then $H$ is a Frölicher-Lie subgroup.

(3) Let $G$ be a Frölicher-Lie group and $H$ a normal (closed) Frölicher-Lie subgroup, then $G / H$ is a Frölicher group and the canonical projection $\pi: G \rightarrow$ $G / H$ is smooth. If $\mathfrak{g} / \mathfrak{h}$ is convenient with lifting property, i.e. a smooth curve to $\mathfrak{g} / \mathfrak{h}$ has a smooth lift along $\pi^{\prime}$ and $G / H$ has the lifting property, then $G / H$ is a Frölicher-Lie group with Lie algebra given by $\mathfrak{g} / \mathfrak{h}$. 
(4) Let $G$ be a Frölicher Lie group and $\mathfrak{h}$ a $c^{\infty}$-closed subalgebra of $\mathfrak{g}$, then there is a unique, smoothly connected, virtual Frölicher-Lie subgroup $H$ with Lie algebra $\mathfrak{h}$.

Remark 6. One might wonder why we do not treat finite or even infinite products of Frölicher-Lie groups, since we developed the whole theory for the infinite dimensional usage. However, since $c^{\infty}\left(G_{1} \times G_{2}\right)$ is in general finer than $c^{\infty} G_{1} \times c^{\infty} G_{2}$, we cannot hope for good compatibility of the product topology and the $c^{\infty}$-topology.

Proof. Let $G$ be a Frölicher-Lie group and $H$ a smoothly connected, closed subgroup, then we can define the future Lie algebra

$$
\mathfrak{h}:=\{X \in \mathfrak{g} \text { such that } \exp (s X) \in H \text { for all } s \in \mathbb{R}\}
$$

as a subset of $\mathfrak{g}$. If $X, Y \in \mathfrak{h}$, then $\exp (s(X+Y))=\lim _{n \rightarrow \infty}\left(\exp \left(\frac{s}{n} X\right) \exp \left(\frac{s}{n} Y\right)\right)^{n}$ lies in $H$ for all times $s$ by closedness and regularity, furthermore $\lambda X \in \mathfrak{h}$ for any real number $\lambda$. Consequently $\mathfrak{h}$ is a linear subspace of $\mathfrak{g}$. By smoothness of the evolution map we obtain its continuity: so $X_{n} \rightarrow X$ in $\mathfrak{g}$ with $X_{n} \in \mathfrak{h}$ leads to $\exp \left(s X_{n}\right) \rightarrow \exp (s X)$ pointwise in the $c^{\infty}$-topology of $G$, consequently $X \in \mathfrak{h}$, so $\mathfrak{h}$ is $c^{\infty}$-closed in $\mathfrak{g}$ and hence convenient. The closed subset $H$ carries the natural structure of a Frölicher group, the metrics can be restricted and so we obtain a Lipschitz-metrizable Frölicher group. Since $\mathfrak{h}$ is convenient and the restriction of the Evolution map is smooth, we are ready.

If $H$ is normal, then $\mathfrak{h}$ is an ideal, since $\operatorname{conj}(g) H=H$ for all $g \in G$, so $A d(g)(\mathfrak{h}) \subset \mathfrak{h}$ for all $g \in G$, therefore $[\mathfrak{g}, \mathfrak{h}] \subset \mathfrak{h}$. We denote $\pi: G \rightarrow G / H$ and $\pi^{\prime}: \mathfrak{g} \rightarrow \mathfrak{g} / \mathfrak{h}$ the projections. We have to define the structure of a Frölicher space on $G / H$ by defining its smooth functions, $f: G / H \rightarrow \mathbb{R}$ is smooth if $f \circ \pi$ is smooth. In particular curves of the type $\pi \circ c$ for $c: \mathbb{R} \rightarrow G$ are smooth, in particular $\pi$ is smooth. The multiplication and inversion are therefore smooth. We investigate now the mapping $\pi_{*} \circ E v o l^{r}: C^{\infty}(\mathbb{R}, \mathfrak{g}) \rightarrow C^{\infty}(\mathbb{R},\{0\} ; G / H,\{H\})$. Given $X: \mathbb{R} \rightarrow \mathfrak{g}$, then $\pi_{*} \circ \operatorname{Evol}^{r}(X)=\pi\left(\operatorname{Evol}^{r}(X)\right)$ only depends on $\pi^{\prime} \circ X$, since any other smooth lift of this curve is a limit of product integrals with exponentials and for exponentials we have

$$
\pi \exp (s(X+Y))=\pi \exp (s X)
$$

for $X \in \mathfrak{g}$ and $Y \in \mathfrak{h}$ by product integration. Consequently we define - via the lifting property for $\mathfrak{g} / \mathfrak{h}$ the evolution map $\widetilde{E v o l^{r}}: C^{\infty}(\mathbb{R}, \mathfrak{g} / \mathfrak{h}) \rightarrow C^{\infty}(\mathbb{R},\{0\} ; G / H,\{H\})$, which is a well defined smooth diffeomorphism.

Next we define the right invariant Lipschitz metrics on $G / H$ via

$$
\widetilde{d_{\alpha}}(g N, h N):=\inf _{n \in N} d_{\alpha}(g n, h)
$$

which provides well-defined right invariant half metrics on $G / H$. They fulfill the Lipschitz-assumptions by definition and the topology is generated by them in the correct sense.

Given finally a $c^{\infty}$-closed subalgebra $\mathfrak{h}$ of $\mathfrak{g}$, then the set

$$
\operatorname{Evol}^{r}\left(C^{\infty}(\mathbb{R}, \mathfrak{h})\right)
$$


is a closed subset of $C^{\infty}(\mathbb{R},\{0\} ; G,\{e\})$, the point evaluations define a subset of $G$, which is a subgroup $H$ by the formula

$$
\begin{gathered}
\operatorname{Evol}^{r}(X) \cdot \operatorname{Evol}^{r}(Y) \\
=\operatorname{Evol}^{r}\left(t \mapsto X(t)+\operatorname{Ad}\left(\operatorname{Evol}^{r}(X)(t)\right) Y(t)\right),
\end{gathered}
$$

which is valid on Frölicher-Lie groups. A curve $c: \mathbb{R} \rightarrow H$ is smooth if it is smooth in $G$, so we get the diffeomorphism property.

Remark 7. The advantage of this setting is two-fold. Charts are obsolete in this setting and analysis is possible due to Lipschitz-metrics and the Frölicher space structure.

\section{REFERENCES}

[1] Andreas Kriegl and Peter W. Michor, The convenient setting of Global Analysis, Mathematical Surveys and Monographs 53, American Mathematical Society, 1997.

[2] Hideki Omori, Infinite-dimensional Lie groups, Translation of Mathematical Monographs 158, American Mathematical Society, Providence, Rhode Island, 1997.

[3] Josef Teichmann, Convenient Hille-Yosida Theory, Revista mathematica complutense, to appear (2002).

[4] Josef Teichmann, A convenient approach to Trotter's formula, Journal of Lie Theory 11, 427 -400 (2001).

[5] _ Infinite dimensional Lie theory from the point of view of functional analysis, Ph.D. thesis, University of Vienna, 1999, directed by Peter Michor.

[6] __ Regularity of infinite-dimensional Lie groups by metric space methods, Tokyo Journal of Mathematics 24, no. 1, $39-58$ (2001).

Institute of financial and actuarial mathematics, Technical University of Vienna, Wiedner Hauptstrasse 8-10, A-1040 Vienna, Austria

E-mail address: josef.teichmann@fam.tuwien.ac.at 